\title{
Whole blood platelet aggregometry in HIV-infected patients on treatment with abacavir ${ }^{*}$
}

\author{
Rosario Palacios Muñoz ${ }^{1 \#}$, Jose A. González-Correa ${ }^{2}$, Josefa Ruiz ${ }^{1}$, Enrique Nuño ${ }^{1}$, Manuel Márquez $^{1}$, \\ Jose P. de la Cruz ${ }^{2}$, Jesús Santos ${ }^{1}$ \\ ${ }^{1}$ Infectious Diseases Unit, Virgen de la Victoria Hospital, Málaga, Spain \\ ${ }^{2}$ Pharmacology Department, Medicine School, Málaga University, Málaga, Spain \\ Email:" $\underline{\text { med006809@saludalia.com }}$
}

Received 16 February 2012; revised 20 March 2012; accepted 20 April 2012

\begin{abstract}
Purpose: To assess platelet activity in HIV-patients with and without antiretroviral therapy (ART), analysing the influence of the presence or absence of ABC in the regimen. Methods: Observational, pilot study, including 30 asymptomatic HIV-patients: 20 on ART for at least 24 weeks and with undetectable HIV viral load-10 on ABC, 10 on tenofovir (TDF)and 10 naïve patients, and a control group of 10 HIV-negative subjects. No subject was receiving drugs with antiagregant activity. Platelet activity was assessed by measuring time-dependent platelet aggregometry (electrical impedance on fasting whole blood), induced by ADP $(1.25,2.5 \mu \mathrm{M})$, collagen $(0.5$, $1 \mu \mathrm{g} / \mathrm{mL})$, arachidonic acid $(100,200 \mu M)$, and U46619 (receptor agonist of the tromboxano $\left.A_{2}\right)(1.25$, $2.5 \mu \mathrm{M})$. Statistic program: SPSS, 16.0. Results: Demographic, anthropometric data, and cardiovascular risk factors were similar in all groups, but older age and longer time of HIV infection in the $A B C$ group (50.4 vs $36.1,34.2$ and 42.7 years, respectively; $p<0.05$, and 140.3 vs 88.1 and 48.3 months in the two other groups of HIV patients; $p<0.05$ ). Mean CD4 cells count was $564 / \mathrm{mm}^{3}$. Platelet aggregation with exposure to U46619 was higher in the ABC compared with the TDF group (11.1 vs $4.4 \%$; $p=0.007)$, naïve patients $(11.1$ vs $5.7 \%$; $p=0.014)$, and the HIV-negative group (11.1 vs $6.5 \% ; p=0.04)$. These differences remained significant when controlled for age and time of HIV infection. Conclusions: ABC increases platelet aggregability possibly in relation with the receptor of tromboxano. Wider studies are needed to confirm this hypothesis.
\end{abstract}

Keywords: Antiretroviral Therapy; Abacavir;

\footnotetext{
*No funding received for this work

No conflict of interest.

${ }^{\#}$ Corresponding author.
}

Cardiovascular Risk; Platelet Activity

\section{INTRODUCTION}

A greater prevalence of cardiovascular risk factors (CVRF) has been seen in patients infected with HIV than in the general population [1,2], with the resulting increase in cardiovascular risk [3-5] and incidence of cardiovascular events [6-8]. Both the HIV infection itself as well as the antiretroviral therapy (ART) seem to play a role in the development of cardiovascular events in these persons $[9,10]$. Although protease inhibitors and thymidine analogues have so far been the drugs most involved in metabolic alterations and cardiovascular disease in HIV-infected patients [4-8], a relation has also been reported between the use of abacavir (ABC) and an increased risk of acute myocardial infarction of up to $90 \%$ [11]. Notwithstanding this, controversy still exists about the association between $\mathrm{ABC}$ and ischaemic heart disease [8,9,12-17], and little is known about the pathogenic mechanism by which $\mathrm{ABC}$ induces a greater risk of coronary heart disease. Platelets are clearly involved in the pathogenesis of atherothrombosis, and accumulating evidence suggests that both an elevated platelet count and platelet hyperreactivity may be associated with adverse cardiovascular events in patients with the acute coronary syndrome $[18,19]$. Platelet aggregation remains the gold standard test to assess platelet activity. This technique involves adding an aggregating agent (e.g., ADP, epinephrine, thrombin, arachidonic acid) to whole blood samples in order to explore some of the main biochemical pathways that are responsible to platelet aggregation, such as thromboxane $\mathrm{A}_{2}$ synthesis (arachidonic acid pathway), ADP activation receptors (namely ADP pathway), phosphoinositide activation (induced with collagen) or the recruitment phenomenon induced through some compounds released in the primary stage of platelet activation (thromboxane receptor activation, calcium, serotonin, etc.) [20]. 
The aim of our study was to analyse platelet aggregation response in stable $\mathrm{HIV}$-infected patients with and without ART and determine the influence of the presence or otherwise of $\mathrm{ABC}$ in the therapeutic regimen.

\section{PATIENTS AND METHODS}

\subsection{Study Design}

We undertook a pilot, cross-sectional observational study of a group of HIV-infected patients regularly followedup at the Infectious Diseases Office of the Virgen de la Victoria Hospital, in Malaga, Spain, and a control group of persons with no HIV infection.

\subsection{Subject Selection}

Among HIV-infected patients over 18 years of age on regular outpatient follow-up, we selected 30 patients: 10 on ART with a regimen that included ABC for at least 12 weeks prior to inclusion in the study, 10 on ART with a regimen that included tenofovir (TDF) for at least 12 weeks prior to inclusion in the study, and 10 patients naïve for ART. Patients attending the office were asked if they would like to participate in the study, provided they fulfilled the inclusion criteria. We selected the first 10 patients who accepted and complied with the inclusion criteria for each group.

Patients were excluded if they had signs of active opportunistic infection or AIDS-defining disease in the month prior to selection, if they were on treatment with non-steroidal anti-inflammatory drugs, antiplatelet aggregators, oral anticoagulants or oral contraceptives, if they had chronic liver disease or a history of any cardiovascular event, as well as women who were pregnant or lactating.

During this same period, we selected 10 age- and sex-matched persons from the general population who were HIV negative and who came from the same hospital catchment area as the HIV-positive patients and who fulfilled the previously described exclusion criteria.

All the participants underwent a physical examination with recording of anthropometric data, measurement of blood glucose and lipid profile after a 12-hour fast, and completion of a questionnaire about cardiovascular risk factors. A common protocol was used to gather the epidemiological, clinical, laboratory and therapeutic parameters of all the patients.

\subsection{Measurement of Platelet Aggregometry}

The main indicator of platelet function was maximum intensity of platelet aggregation induced by suboptimal concentrations of ADP, collagen, arachidonic acid and the thromboxane-endoperoxides receptor agonist U46619 , in whole blood, as measured by electrical impedance
[21] with a Chrono-Log 540S aggregometer (ChronoLog Corp., Haverton, PA, USA). Sodium citrate (3.8\%) at a proportion of 1:10 was used as the anticoagulant for samples of whole blood. The samples were incubated for $5 \mathrm{~min}$ at $37^{\circ} \mathrm{C}$, then 1.25 and $2.5 \mu \mathrm{M} \mathrm{ADP}, 0.5$ and 1 $\mu \mathrm{g} / \mathrm{mL}$ collagen, 100 and $200 \mu \mathrm{M}$ arachidonic acid or 1.25 and $2.5 \mu \mathrm{M}$ U46619, were added. The changes in electrical impedance (ohms) were recorded, and maximum intensity of aggregation was considered the maximum change in impedance $10 \mathrm{~min}$ after the inducing agent was added.

\subsection{Statistical Analysis}

The dependent variable was the maximum intensity of platelet aggregation (ohms). The continuous variables are expressed as the median and interquartile range and the qualitative variables as absolute numbers and percentages. For the multivariate analysis on the outcome variable, the starting hypothesis was that $\mathrm{ABC}$ was associated with greater platelet aggregability. Thus, an analysis of variance and the Tukey multiple comparison test were carried out, or the Kruskal-Wallis test if the requirements for use of the ANOVA were not fulfilled. The data were analyzed with SPSS version 16.0 (SPSS software, Chicago, IL), under licence to the Central Computer Service of Malaga University.

\subsection{Ethics}

The study was approved by the Ethics and Research Committee of Virgen de la Victoria Hospital. All the patients were informed of the nature of the study and gave their consent. At all times patient confidentiality and data were respected, in accordance with current legislation (Ley Orgánica 15/1999 de Datos de Carácter Personal).

\section{RESULTS}

The demographic and anthopometric characteristics, the prevalence of CVRF and the lipid and blood glucose profiles were all similar in the four study groups, except that the patients in the ABC group were older (50.4 vs. $36.1,34.2$ and 42.7 years, respectively; $p<0.05)$ and had had HIV for longer (140.3 vs. 88.1 and 48.3 months in the other two HIV groups; $p<0.05$ ) (Table 1). The mean CD4 lymphocyte count in the HIV patients was 564 per $\mathrm{mm}^{3}$.

Maximum intensity of platelet aggregation was not statistically different between negative HIV and HIV naïve groups $(\mathrm{p}>0.2)$ (Figures 1(a-d)). Patients on ABC treatment showed a statistical higher platelet aggregation response to U46619 than naïve patients ( $\mathrm{p}=$ 0.014 with $1.25 \mu \mathrm{M}$ and $\mathrm{p}=0.032$ with $2.5 \mu \mathrm{M}$ ) and negative HIV patients $(\mathrm{p}<0.02)$ (Figure 1(d)). Moreover these patients showed higher values of maximum 
Table 1. Characteristics of the 40 subjects included in the study and comparison between groups.

\begin{tabular}{|c|c|c|c|c|c|}
\hline Variables & HIV-negative & HIV naïve & HIV on ABC & HIV on TDF & $\mathbf{p}$ \\
\hline Male sex & $6(60)$ & $8(80)$ & $10(100)$ & $8(80)$ & 0.3 \\
\hline Age (years) & $42.7(35.7-50.1)$ & $34.2(27.6$ - 41.9) & $50.4(45.7-52.0)$ & $36.1(30.1$ - 44.9) & 0.0001* \\
\hline BMI $\left(\mathrm{kg} / \mathrm{m}^{2}\right)$ & $27.0(20.9-34.4)$ & $25.9(21.8-31.8)$ & $24.6(23.0-26.7)$ & $24.5(20.5-27.0)$ & 0.6 \\
\hline Waist (cm) & $98.5(82-118)$ & $87.4(78-99)$ & $88.5(83-94)$ & $83.2(72-91)$ & 0.07 \\
\hline Tobacco & $2(20)$ & $3(30)$ & $5(50)$ & $6(60)$ & 0.3 \\
\hline Months since HIV diagnosis & - & $48.3(5.0-58.3)$ & $140.3(124.2$ - 170.4) & 88.1 (53.7 - 109.5) & $0.009 *$ \\
\hline Mean CD4 (cel/ $\mu \mathrm{L})$ & - & $590(393-787)$ & $509(361-609)$ & $598(477-704)$ & 0.4 \\
\hline Glycemia (mg/dl) & $94(85-98)$ & $94(84-106)$ & $102(93-106)$ & $98(92-105)$ & 0.2 \\
\hline Triglycerides (mg/dl) & $94(62-126)$ & $119(77-193)$ & $162(103-183)$ & $131(82-168)$ & 0.1 \\
\hline Cholesterol (mg/dl) & $200(188-215)$ & $193(172-216)$ & $229(204-272)$ & $193(171-222)$ & 0.053 \\
\hline HDL-cholesterol (mg/dl) & $60(53-68)$ & $45(40-50)$ & $58(42-66)$ & $45(36-51)$ & 0.07 \\
\hline LDL-cholesterol LDL (mg/dl) & $122(102-131)$ & $122(104-140)$ & $124(91-156)$ & $113(102-129)$ & 0.8 \\
\hline Hypertension & 0 & 0 & $3(30)$ & 0 & 0.03 \\
\hline Family history of CHD & $1(10)$ & $2(20)$ & $2(20)$ & 0 & 0.4 \\
\hline Cardiovascular risk (\%) & $3.28(1-4.5)$ & $4(1-6.2)$ & $9.18(5-13)$ & $4.5(1-8.5)$ & $<0.05^{* *}$ \\
\hline
\end{tabular}

ABC: abacavir. TDF: tenofovir. BMI: body mass index. CHD: coronary heart disease. *Between patients on ABC compared with those on TDF and naïve. **Between patients on ABC compared with those on TDF, naïve and HIV-negative subjects. Quantitative variables are expressed as mean (IQR), and qualitative variables as $\mathrm{n}(\%)$.

intensity of U46619-induced platelet aggregation than TDF patients $(\mathrm{p}=0.007$ with $1.25 \mu \mathrm{M})$ (Figure 1(d)). These differences remained after controlling for age and duration of HIV.

\section{DISCUSSION}

The results of this study demonstrate greater platelet aggregability in the presence of $\mathrm{ABC}$ mediated by the platelet membrane thromboxane-endoperoxides receptors. The greater cardiovascular risk and incidence of cardiovascular events in HIV-infected patients is explained by a greater prevalence of CVRF in this population, together with the inflammatory effect of HIV itself and the metabolic alterations associated with antiretroviral therapy [1-10]. ABC has always been considered one of the more benign antiretroviral drugs, with a better metabolic profile than other nucleoside analogues [3,8,11,17,22,23]. Thus, the need to search for a pathogenic mechanism to account for the possible association between $\mathrm{ABC}$ and ischaemic heart disease suggested in certain cohort studies $[8,11,13]$. Additionally, the reversibility of the greater coronary risk associated with $\mathrm{ABC}$ suggests that it is due to a direct effect of the drug on atherogenesis, in which platelets play a fundamental role. In a study to determine platelet function in 58 patients on ART, Satchell et al. [24] noted platelet hyperreactivity in the presence of ADP, collagen and epinephrine in the patients on $\mathrm{ABC}$, suggesting that this mechanism was responsible for the association between $\mathrm{ABC}$ and ischaemic heart disease. These same authors, in a more recent case-control study, detected that the platelets in HIV-positive patients were more reactive to epinephrine, but less reactive to collagen, ADP and the thrombin receptor-activating peptide, unlike the platelets in HIV-negative persons [25]. These data coincide with findings from a German study of HIV-infected men on tipranavir-containing HAART that demonstrated decreased ADP and collagen-induced platelet aggregation after doses of tipranavir [26]. These results were confirmed in vitro in blood from healthy volunteers exposed to increasing concentrations of tipranavir. In our study, we found no differences in platelet aggregability induced by ADP and collagen between the various groups, though the methodology used differs to that of earlier studies. In our analysis we included two thromboxane-related inducers, arachidonic acid, for which no differences were found between the groups, and U46619, a thromboxane-endoperoxides receptor agonist. With this agonist we observed greater platelet aggregability in the patients on ART with ABC as compared with the patients on ART with TDF, naïve patients and non-HIV persons. The thromboxane-related platelet aggregation is irreversible and, furthermore, its 
ADP

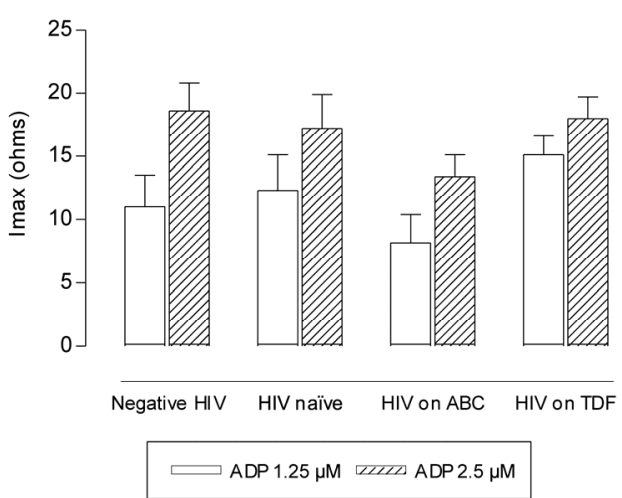

(a)

ARACHIDONIC ACID

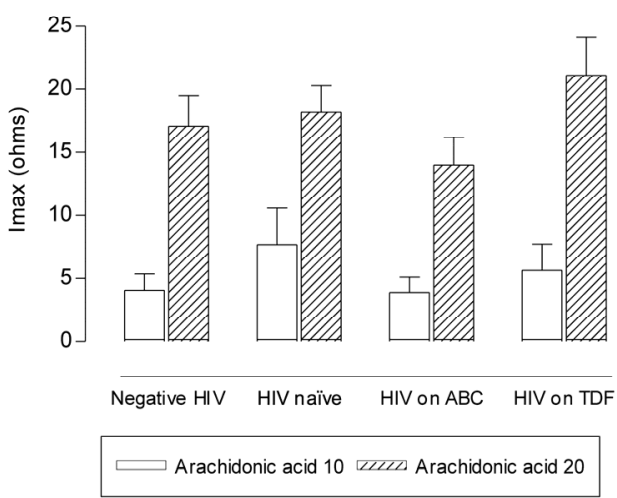

(c)

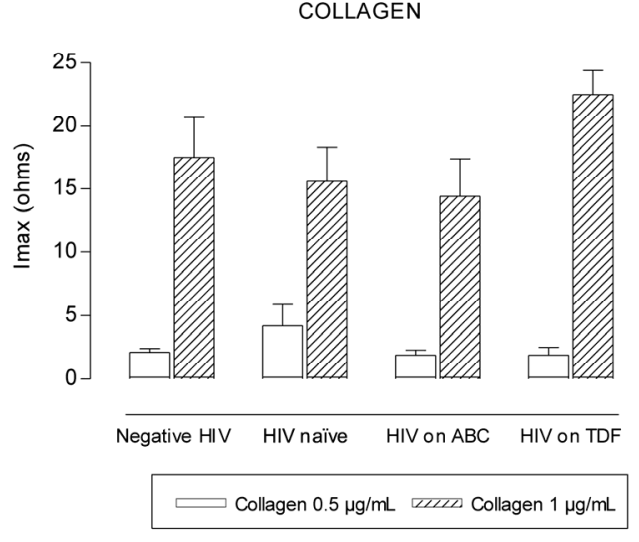

(b)

U46619

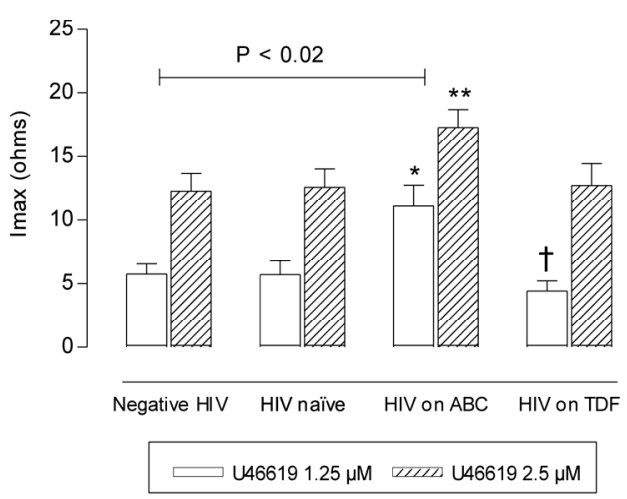

(d)

Figure 1. Maximum intensity (Imax) of platelet aggregation in whole blood induced with ADP, collagen, arachidonic acid and U46619, from negative HIV, HIV naïve, HIV on abacavir (ABC) or tenofovir (TDF) treatment. ${ }^{*} \mathrm{p}=0.014$, $* * p=0.032$, with respect to naïve group. $\uparrow \mathrm{p}=0.007$ with respect to $\mathrm{ABC}$ group.

role in platelet activation is not only due to its direct effect on these cells, but also to a potent vasoconstrictor effect, amplifying the thrombotic response induced by ADP, collagen or epinephrine [20].

Our study has certain limitations, such as the reduced number of cases and the cross-sectional design, which does not therefore permit establishment of an association with cause.

Nevertheless, the results suggest an increase in platelet aggregability in the presence of $\mathrm{ABC}$, at least in relation with the TxA2 receptor activation. Larger, prospective clinical studies are needed, as well as in vitro analysis, to clarify the complexity of the platelet function in these patients and the potential role of $\mathrm{ABC}$ in platelet hyperreactivity.

\section{REFERENCES}

[1] Santos, J., Palacios, R., González, M., Ruiz, J. and Márquez, M. (2005) Atherogenic lipid profile and cardiovascular risk factors in HIV-infected patients (Nétar
Study). International Journal of STD \& AIDS, 16, 677680.

[2] Jericó, C., Knobel, H., Sorli, M.L., Montero, M., Guelar, A. and Pedro-Botet, J. (2006) Prevalencia de factores de riesgo cardiovascular en pacientes con infección por el VIH. Revista Clínica Española, 206, 556-559.

[3] Friis-Møller, N., Reiss, P., Sabin, C.A., et al., Data Collection on Adverse Events of Anti-HIV Drugs (DAD) Study Group (2007) Class of antiretroviral drugs and the risk of myocardial infarction. The New England Journal of Medicine, 356, 1723-1735. doi:10.1056/NEJMoa062744

[4] Holmberg, S.D., Tong, T.C., Ward, D.J., et al., HIV Outpatient Study (HOPS) (2002) Protease inhibitor drug use and adverse cardiovascular outcomes in ambulatory HIV-infected persons. Lancet, 360, 1747-1748. doi:10.1016/S0140-6736(02)11672-2

[5] Mary-Krause, M., Cotte, L., Simon, A., et al., Clinical Epidemiology Group from the French Hospital Database (2003) Increased risk of myocardial infarction with duration of protease inhibitor therapy in HIV-infected men. AIDS, 17, 2479-2486. doi:10.1097/00002030-200311210-00010 
[6] Grinspoon, S., Grunfeld, C., Kotler, D.P., Currier, J.S., Lundgren, J.D., Dubé, M.P., et al. (2008) State of the science conference: Initiative to decrease cardiovascular risk and increase quality of care for patients living with HIV/AIDS: Executive summary. Circulation, 118, 198210. doi:10.1161/CIRCULATIONAHA.107.189622

[7] D’Arminio, A., Sabin, C.A., Phillips, A.N., Reiss, P., Weber, R., Kirk, O., et al. (2004) Cardio- and cerebrovascular events in HIV-infected persons. AIDS, 18, 18111817. doi:10.1097/00002030-200409030-00010

[8] Worm, S.W., Sabin, C., Weber, R., Reiss, P., El-Sadr, W., Dabis, F., et al. (2010) Risk of myocardial infarction in patients with HIV infection exposed to specific individual antiretroviral drugs from the 3 major drug classes: The data collection on adverse events of anti-HIV drugs (D: A:D) study. The Journal of Infectious Diseases, 201, 318330. doi:10.1086/649897

[9] Baker, J.V., Neuhaus, J., Duprez, D., et al., for the INSIGHT SMART Study Group (2011) Changes in inflammatory and coagulation biomarkers: A randomized comparison of immediate versus deferred antiretroviral therapy in patients with HIV infection. The Journal of Infectious Diseases, 56, 36-43.

[10] Calmy, A., Gayet-Ageron, A., Montecucco, F., et al., STACCATO Study Group (2009) HIV increases markers of cardiovascular risk: Results of a randomized, treatment interruption trial. AIDS, 23, 929-939. doi:10.1097/QAD.0b013e32832995fa

[11] Sabin, C.A., Worm, S.W., Weber, R., et al., D:A:D Study Group (2008) Use of nucleoside reverse transcriptase inhibitors and risk of myocardial infarction in HIV-infected patients enrolled in the D:A:D study: A multi-cohort collaboration. Lancet, 371, 1417-1426. doi:10.1016/S0140-6736(08)60423-7

[12] Marin, A., Amin, J., Cooper, D., et al., STEAL Study Group (2010) Abacavir does not affect circulating levels of inflammatory or coagulopathic biomarkers in suppressed HIV: A randomized clinical trial. AIDS, 24, 2657-2663. doi:10.1097/QAD.0b013e32833f147f

[13] Lang, S., Mary-Krause, M., Cotte, L., et al., Clinical Epidemiology Group of the French Hospital Database on HIV (2010) Impact of individual antiretroviral drugs on the risk of myocardial infarction in human immunodeficiency virus-infected patients: A case-control study nested within the French Hospital Database on HIV ANRS cohort CO4. Archives of Internal Medicine, 170, 12281238. doi:10.1001/archinternmed.2010.197

[14] Brothers, C.H., Hernández, J.E., Cutrell, A.G., Curtis, L., Ait-Khaled, M., Bowlin, S.J., et al. (2009) Risk of myocardial infarction and abacavir therapy: No increased risk across 52 GlaxoSmithKline-sponsored clinical trials in adult subjects. Journal of Acquired Immune Deficiency Syndromes, 51, 20-28.

doi:10.1097/QAI.0b013e31819ff0e6

[15] Benson, C., Ribaudo, H., Zheng, E., et al. (2009) No as- sociation of abacavir use with risk of myocardial infarction or severe cardiovascular disease events: Results from ACTG A5001. Program and Abstracts of the 16th Conference on Retroviruses and Opportunistic Infections, Montreal, 8-11 February 2009,Abstract 721.

[16] Costagliola, D., Lang, S., Mary-Krause, M. and Boccara, F. (2010) Abacavir and cardiovascular risk: Reviewing the evidence. Current HIV/AIDS Reports, 7, 127-133. doi:10.1007/s11904-010-0047-3

[17] Behrens, G.M. and Reiss, P. (2010) Abacavir and cardiovascular risk. Current Opinion in Infectious Diseases, 23, 9-14. doi:10.1097/QCO.0b013e328334fe84

[18] Daví, G. and Patrono, C. (2007) Platelet activation and atherothrombosis. The New England Journal of Medicine, 357, 2482-2494. doi:10.1056/NEJMra071014

[19] Kottke-Marchant, K. (2009). Importance of platelets and platelet response in acute coronary syndromes. Cleveland Clinic Journal of Medicine, 76, S2-S7. doi: $10.3949 /$ ccjm.76.s1.01

[20] Li, Z., Delaney, M.K., O’Brien, K.A., et al. (2010) Signaling during platelet adhesion and activation. Arteriosclerosis, Thrombosis, and Vascular Biology, 30, 23412349. doi:10.1161/ATVBAHA.110.207522

[21] Cardinal, D.C. and Flower, R.J. (1980) The electronic aggregometer: A novel device for assessing platelet behaveior in blood. Journal of Pharmacological Methods, 3, 135-158. doi:10.1016/0160-5402(80)90024-8

[22] Moyle, G.J., Sabin, C.A., Cartledge, J., et al., RAVE (Randomized Abacavir versus Viread Evaluation) Group UK (2006) A randomized comparative trial of tenofovir DF or abacavir as replacement for a thymidine analogue in persons with lipoatrophy. AIDS, 20, 204-250. doi:10.1097/01.aids.0000247574.33998.03

[23] Jones, R., Sawleshwarkar, S., Michailidis, C., Jackson, A., Mandalia, S., Stebbing, J., et al. (2005) Impact of antiretroviral choice on hypercholesterolaemia events: The role of the nucleoside reverse transcriptase inhibitor backbone. HIV Medicine, 6, 396-402. doi:10.1111/j.1468-1293.2005.00325.x

[24] Satchell, C., O’Connor, E., Peace, A., et al. (2009) Platelet Hyper-Reactivity in HIV-1-infected Patients on Abacavir-containing ART. Program and Abstracts of the 16th Conference on Retroviruses and Opportunistic Infections, February 8-11 2009, Montreal, Canada, Abstract 151LB.

[25] Satchell, C.S., Cotter, A.G., O’Connor, E.F., Peace, A.J., Tedesco, A.F., Clare, A., et al. (2010) Platelet function and HIV: A case-control study. AIDS, 24, 649-657. doi:10.1097/QAD.0b013e328336098c

[26] Graff, J., Von Hentig, H., Kuczka, K., Angioni, C., Gute, P., Klauke, S., et al. (2008) Significant effects of tipranavir on platelet aggregation and thromboxane B2 formation in vitro and in vivo. Journal of Antimicrobial Chemotherapy, 61, 394-399. doi:10.1093/jac/dkm486 